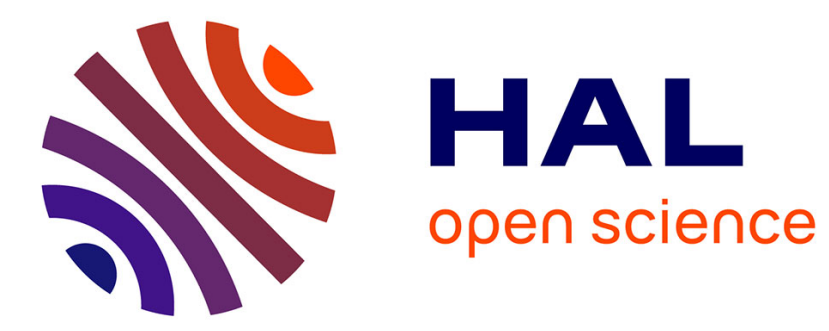

\title{
L'inscription dédicatoire du théâtre du Bois l'Abbé à Eu (Seine-Maritime)
}

\author{
Michel Mangard
}

\section{To cite this version:}

Michel Mangard. L'inscription dédicatoire du théâtre du Bois l'Abbé à Eu (Seine-Maritime). Gallia - Fouilles et monuments archéologiques en France métropolitaine, 1982, 40 (1), pp.35-51. 10.3406/galia.1982.1852 . hal-01940266

\section{HAL Id: hal-01940266 https://hal.science/hal-01940266}

Submitted on 27 Feb 2020

HAL is a multi-disciplinary open access archive for the deposit and dissemination of scientific research documents, whether they are published or not. The documents may come from teaching and research institutions in France or abroad, or from public or private research centers.
L'archive ouverte pluridisciplinaire HAL, est destinée au dépôt et à la diffusion de documents scientifiques de niveau recherche, publiés ou non, émanant des établissements d'enseignement et de recherche français ou étrangers, des laboratoires publics ou privés.

\section{(ㅇ)(1) $\$$}

Distributed under a Creative Commons Attribution - NonCommercial - NoDerivatives| 4.0 


\title{
L'INSCRIPTION DÉDICATOIRE DU THÉÂTRE DU BOIS L'ABBÉ À EU (Seine-Maritime)
}

\author{
par Michel MANGARD
}

A $4 \mathrm{~km}$ environ au sud sud-est dr la ville d'ku (arr. de Dieppe) s’élève entre la vallée de la Breste au nord nord-est et te vallon de Saint-Pierre-en-Val au sud-ouest une série de croupes plus ou moins boisées, séparées par de brèves dépressions, formant la partie du triège d Eu appelée Bois l A bbé (lig. 1). C"est dans le secteur du canton forestier dénommé Le Cirque que fut partiellement remis au jour entre 1965 et 1973 un théatre déjà reconnu

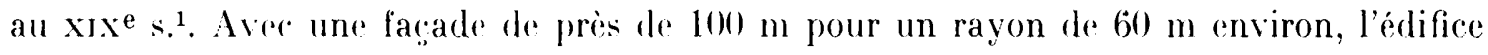
compte parmi les plus vastes de sa catégorie en Haute-Normandie ${ }^{2}$.

En avant du frons scaenae orienté nord-sud et mesurant 13,26 $\mathrm{m}$ se dressaient origrinellement cinq colonnes a cannelures rudentées, ornées de feuilles imbriquées et de reliefs. Leur ruine entraina la chute d'une inseription dédicatoire (fig. : I) et 3) dont en 1965) nous recueillimes 40 fragments portant le texte suivant:

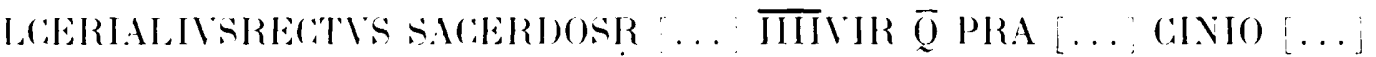

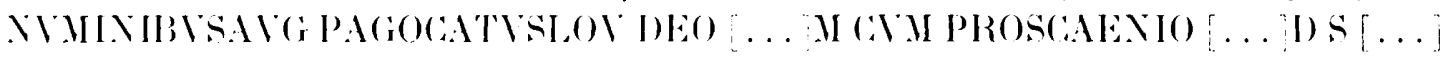

L'inscription entiire se développait sur six plaques dont nous avons reconstitué completement

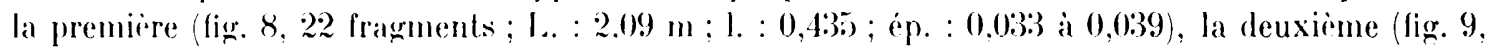

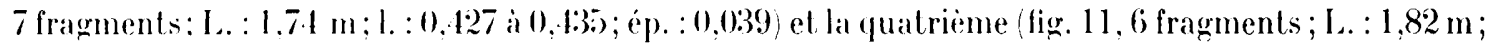
1. : 0.429 a 0.417 ; ép. max. : 0,056). De la troisieme plaque (lig. 10) subsistent trois fragments de l'exlrémité droite (ép. moy: :0,041 de la sixime, deux fragments du début (fig. $12 ;$ I. max.

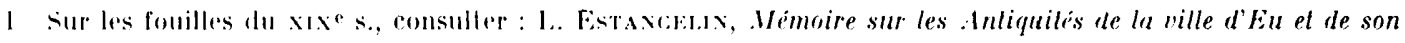

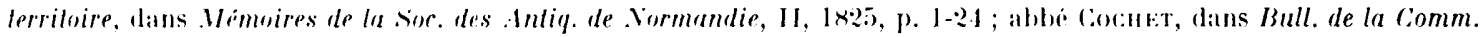

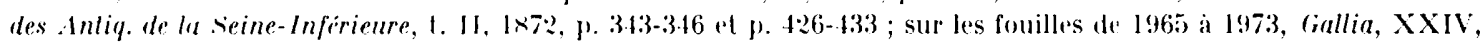

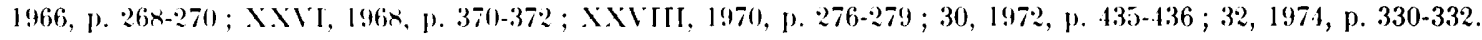

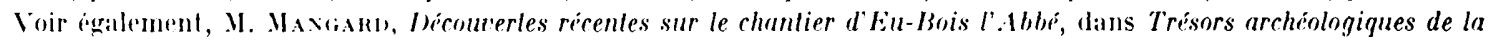
Haule-Normandie, Rouen, 1980, p. 1:21-1:29.

$\because$ C. Vanogleal'x, Les édifices théalraux gallo-romains de Normandie, Hourn, 1979. 


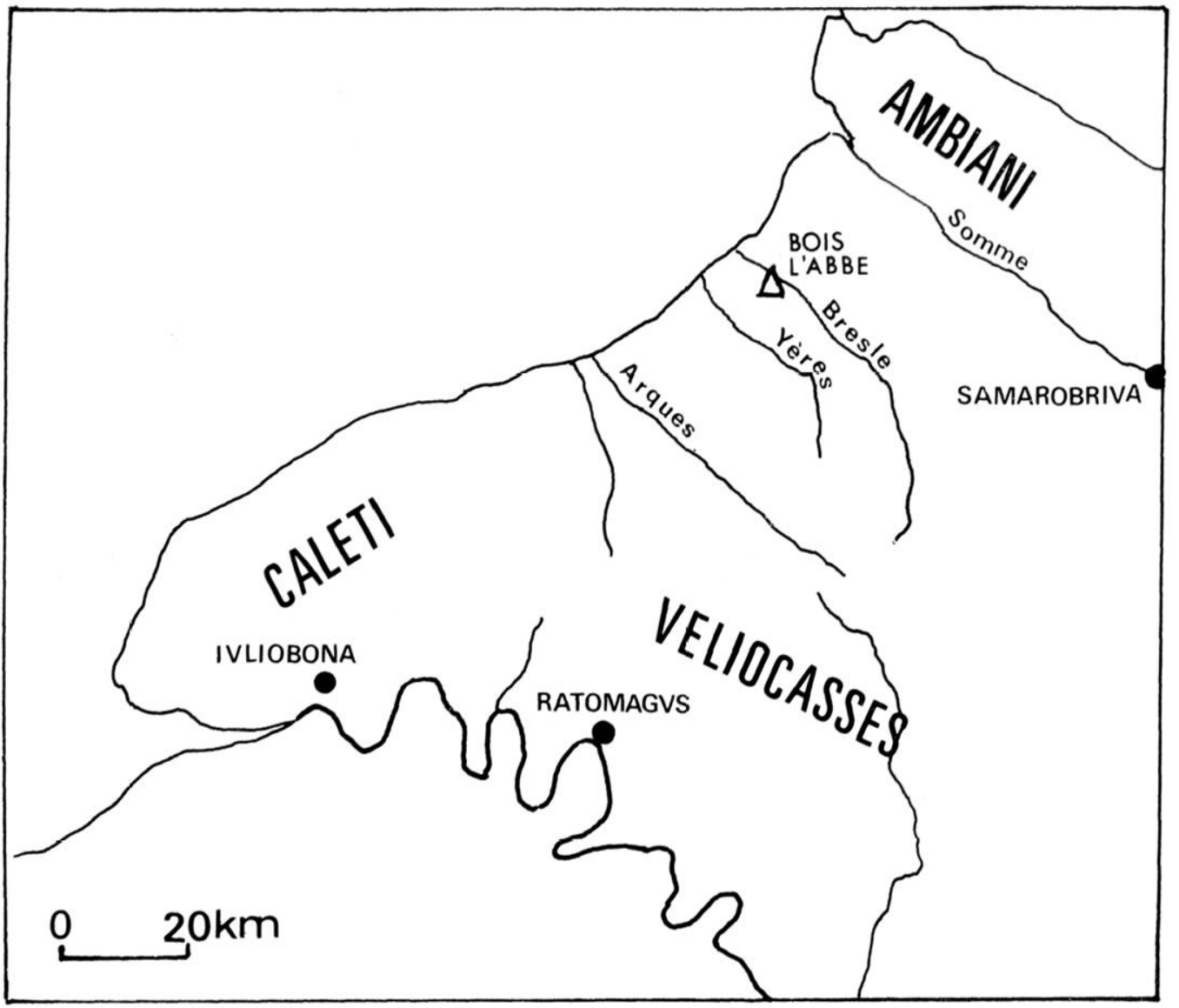

1 Carte de silualion du sile du Bois l'Mbhe.
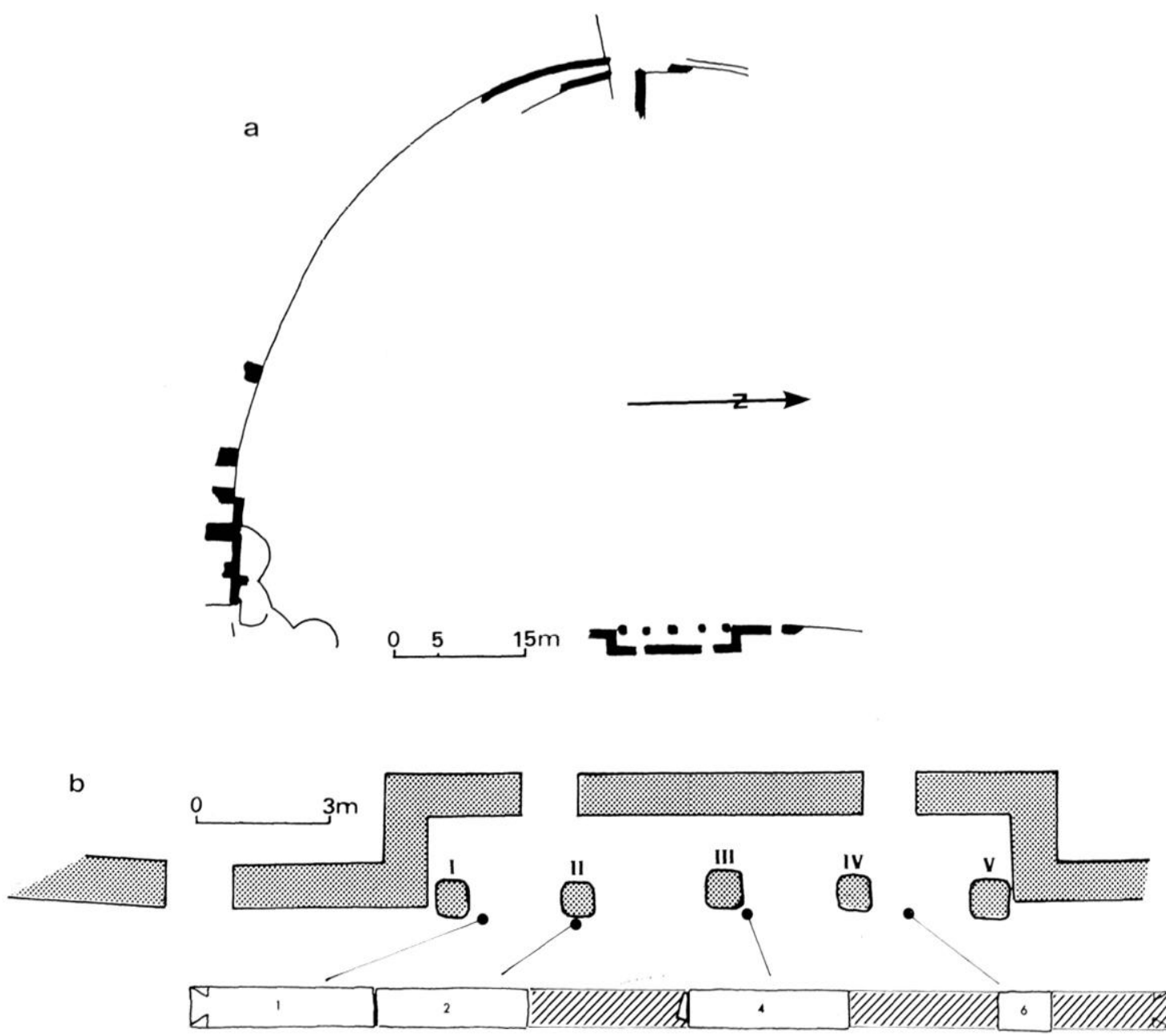


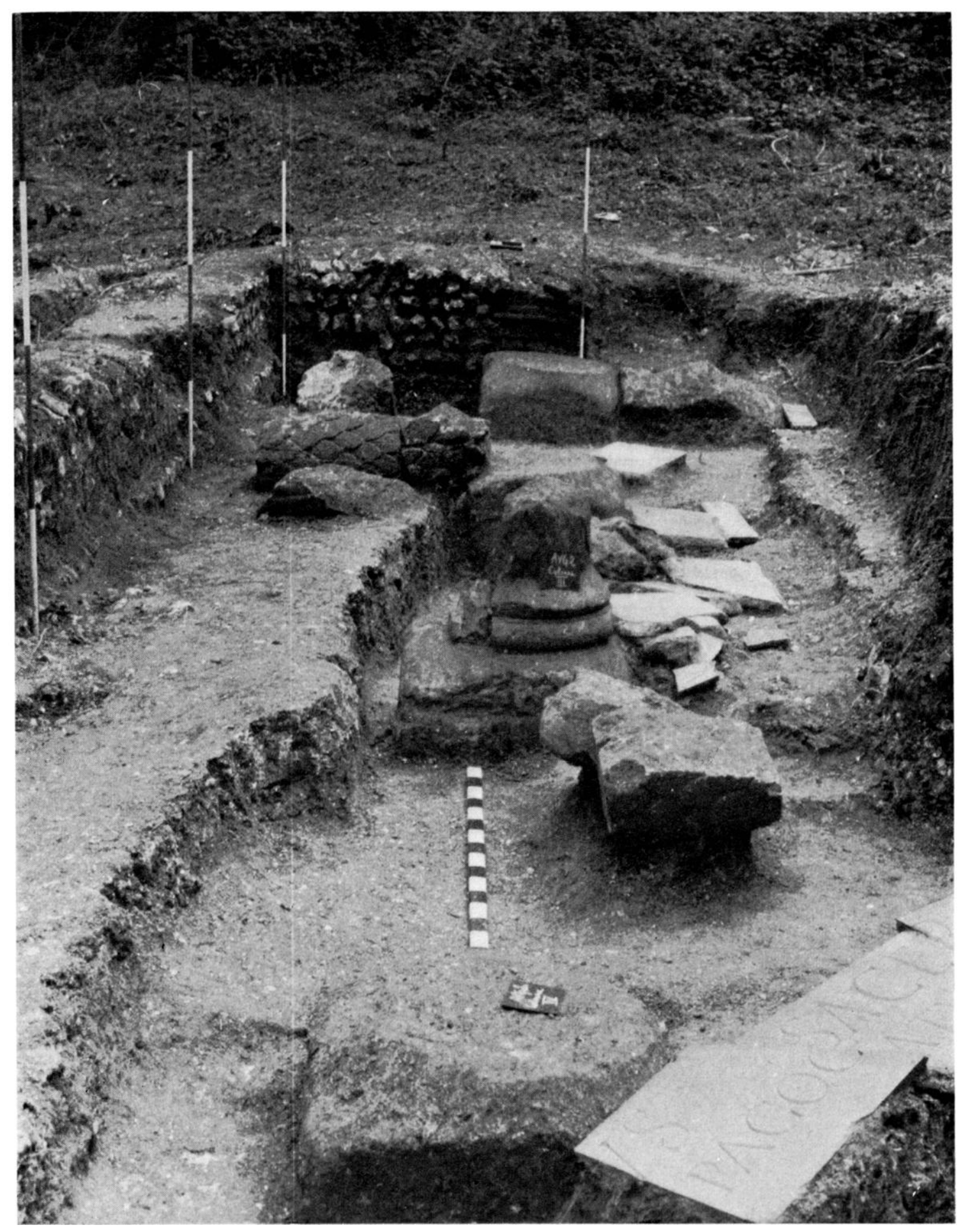

3 l.e proscuenium en cours de fouille, vu du nord; au premier plan, la plaque 2. 
conservée : $0,59 \mathrm{~m} ; 1$. : 0,$432 ;$ ép. : 0,044 à 0,050$)$. Rien n'a été retrouvé de la cinquiène plaque ${ }^{3}$. L'ensemble étail fixé par des crampons de fer à une poutre, sinon à une architrave en bois. Les figures 4 a 7 montrent la disposition des plaques lors de la découverte.

Le texle a été gravé sur deux lignes. Les lettres de la première mesurent en moyeme $13 \mathrm{~cm}$ de hauteur, celles de la seconde un peu moins de $10 \mathrm{~cm}$. Malgré la beauté évidente d'une gravure soignée, on observe quelques irrégularités ; la taille des lettres. comme la largeur des plaques, tend à diminuer légèrement de la gauche vers la droite; on ne voit aucun tracé de ligne directrice. Lne queue-d'aronde -- conservée sur la première plaque. ornait chaque extrémité de l'inscription. Plusieurs lettres de la quatrième plaque présentent encore des traces d'un enduit blanc qui servait de couche de fond à la peinture rouge faisant ressorlir le texte. Il n'y a ni ligature, ni interpunction; les lettres de chaque partie du texte (Iria nomina, éléments du cursus, etc.) forment un ensemble séparé du suivant par un vide d'une trentaine de cm sur la première ligne et d'une vinglaine sur la serende qui commence en retrait de $28 \mathrm{~cm}$.

Yous proposons de compléter et de développer l'inscription comme suit :

L(ucius) Cerialius Rochus, sacerdos R amae el Aug(usli), IIII vir, q(uaeshor). pralefeclus lalro]cinio [arcendo (?) ] Numinibus Aug(uslorum), pago Caluslou(go). deo :Marli thealru]m cum proscaenio [et suis ornamenlis]d(e) s(ua) [p(ecunia) fecil.

La restitution $R$ [omae et $A u g(u s t i)$ sur la plaque 3 nous parait certaine. Le début de la lettre conservée a la fin de la deuxième plaque (fig. 9) peut appartenir à un P' ou a un R. Une formule du type sacerdes Provinciae... est etrangère a la Ciaule Chevelue ; par contre, sacerdos Romae el Aug(usli) est bien attesté. Compte tenu de la restitution du texte, la plaque 3 mesurerait ainsi un peu plus de $1,75 \mathrm{~m}$ (fig. 1:3) ${ }^{6}$. A la seconde ligne, Ie .I final conservé et le contexte suggèrent le complément flhealru'm précédé d'un vide; l'espace restant disponible convient pour un théonyme au datif, de cing ou six lettres. pour lequel nous proposons Marli.

La conjecture pralefeclus lalro cinio fareendo donne à la plaque is une longueur comprise entre 1,60 et 1,65) an à la plaque 6 une longueur complete identique à celle de la première?. Pour la deuxième ligne lue fel suis arnamentis d(e) s(ua) (p(ecunia) fecil:

3 Indipendamment du contemu du texte, la position sur le terain permet deblablir avec surete le nombre et l'ordre des plaques. Ides fragments de la plaque 1 ont ete recueitlis depuis le dé de la colonne 1 jusqua proximité

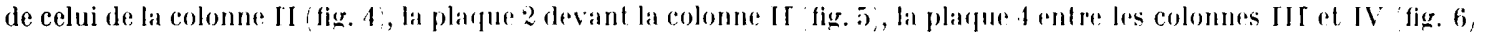

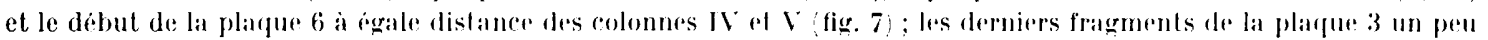

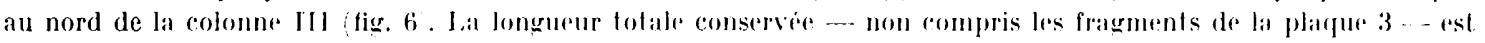
de $6,24 \mathrm{~m}$.

4 Au nombre d'au moins qualre par plargue, ils mesurent de lx à :al com.

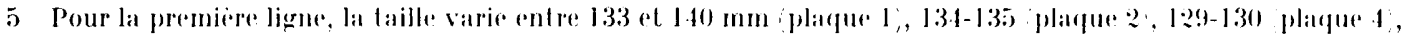

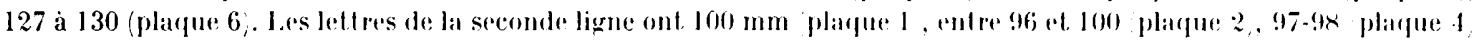
et 98 plaque 6 ; ; le .11 de la playue 3 mesure 95 mm.

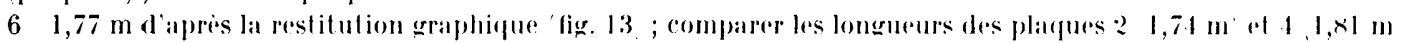
intégralement conservés.

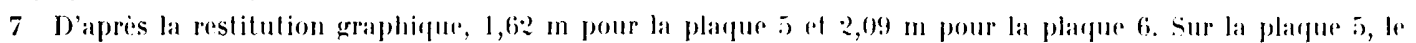
texte de la deuxieme ligne doit oecuper loute la longuene de la pierre ; il y a done place pour 1.4 a 16 lettres selon la largeur de celles-ci. Ie complement el suis columnis, I.L.s. 5jos, aurfuel on pouvait penser d'apres rum proscaenio 

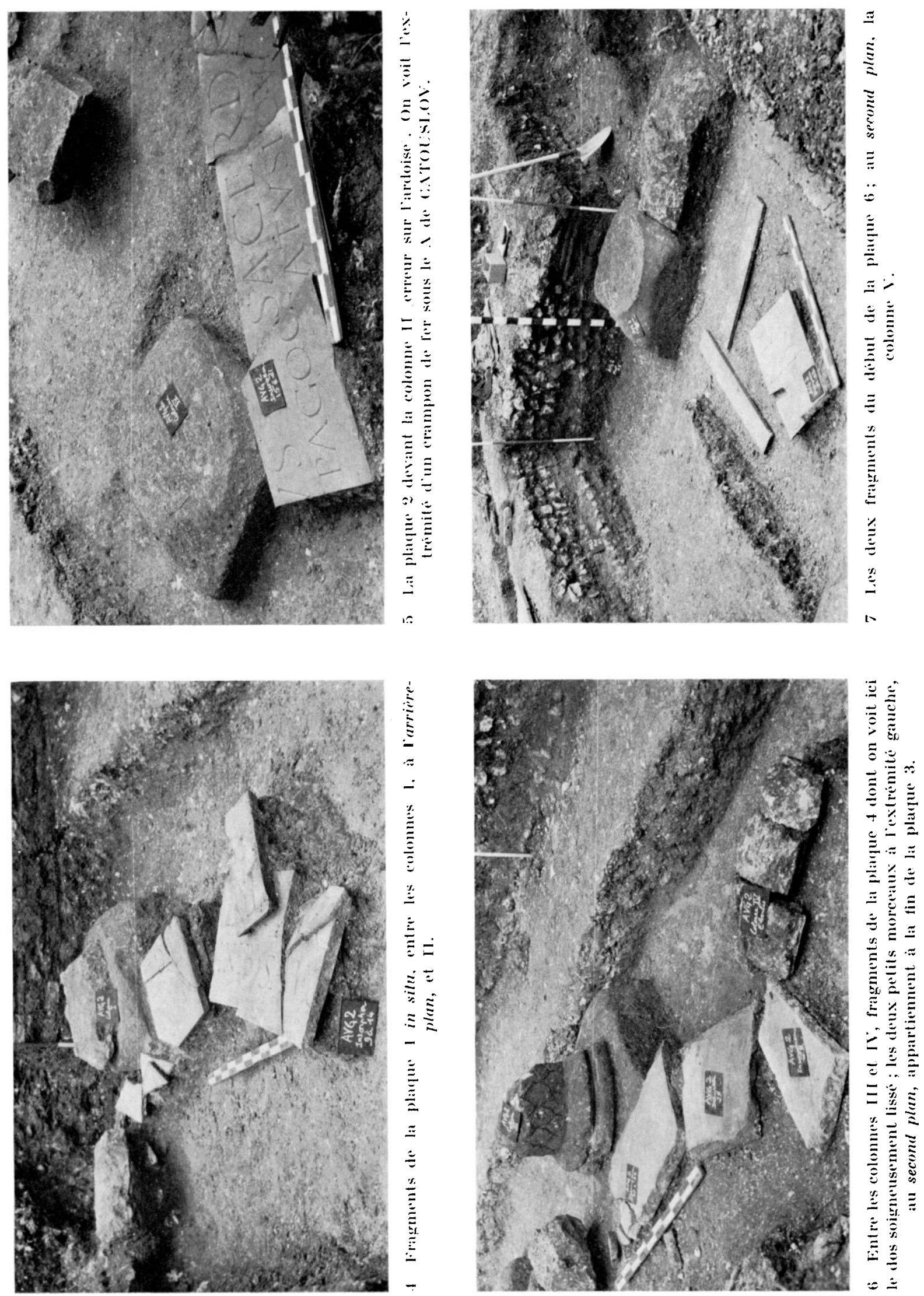
le choix antre les restitutions possibles tient compte de ces longururs hypothétiques at de la présentation du texte. Ainsi restituée. l'inscription mesure plus de $11 \mathrm{~m}^{8}$; rappelons que: la distance entre axes de la colonne I à la colonne $V$ est de 12.25$) \mathrm{m}$.

La dédieace numinibus .lug., à lire numinibus Augustorum ${ }^{9}$, offre, compte tenu du contexte archéologique, une fourchette de datation entre 161 et $211^{10}$. La facture des leltres ratlache également l'inscription à des modèles d'écriture monumentale que l'on rencontre dans tout le monde romain sur les dédicaces officielles d'exécution soignée, depuis les Intonins jusqu'aux sévères ${ }^{11}$. Le décor d'imbrications des colonnes, contemporaines de l'inscription ${ }^{12}$, ne contredit pas cette chronologie large qu'il est cependant possible de serrer davantage. Les monnaies les plus récentes trouvées dans les terres rapportées pour les remblais de conslruction dans la carea et dans le proscaenium sont deux sesterces de Lucius Vérus (Rome, 164, R.I.C., 1:370) et de Narc-Aurèle (Rome, entre 166 et 168 , R.I.C., 948 ou 9:99); à en jugrer par leur degré d'usure, ils ont été perdus bien après leur date d'émission el excluent une datation remontant a l'association Marc-Auriale - Vérus. (161-169). En revanche, dans les niveaux de fréquentation et d'abandon de l'édifice. hormis la céramique réduite à quelques tessons sans signification, le matériel datable apparlient tout entier

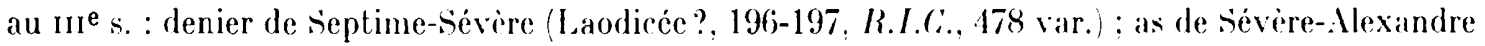
(Rome, 22t, R.I.C., 569), antoniniani de Claude II (Kome, 268, R.I.C., 279) et d'Aurélien (Milan. 271-274, R.I.C., 128). Le bon état de conservation du denier, qui a peu circulé, nous inclinerail i dater des règnes conjoints de septime-sévere et de Caracalla, entre 198 el $211^{13}$. l'inscription et les: travaux qui en furent l'occasion. I'autre part, si l'on considere que d'apris les observations straligraphiques la construction du grand temple est à dater également de la fin du me - début du me s. et que ce qui subsiste de son décor architectural s'accorde bien au goùt sévérien, nous pouvons sans doute attribuer aux premières années de la nouvelle dynastie l'activité déployée au Bois l'Abbé ${ }^{14}$.

Conforme a la trarlition, ou plutiot à l'obligation d'évergétisme des notables muni(ipaux ${ }^{15}$, la construction du théatre devait représenter une lépense importante dans la

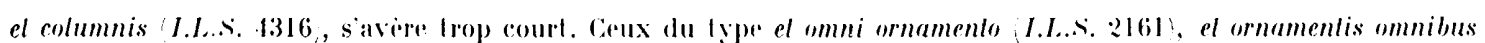
I.L..S. 9364), concevables à partir de proscaenium el orchestram cum ornamentis (I.l..t. 5640), sont lrop longs. Nous avons opte pour et suis ornamentis sur le modele de la formule cum suis ornamentis tris couramment attestere la fin du texte est commune; pour I) S P, voir la dédicace du thealro de leours XIII, 1642 I.L... 56.39.

$\times \quad 11,1 \% \mathrm{~m}$ selon la restitution graphique.

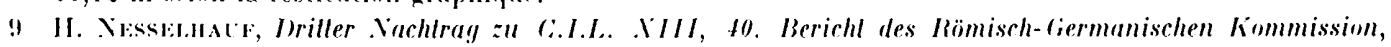
l9:9, p. 133, considere numinibus dug(ustorum) comme geniemalement plus valisemblable que muminibus Aug(ustis). J) Ia dedicace est faite probablement aux empereurs vivants. Dans l'etat actuel de ba documentation, l'emploi de deo devant un nom de divinite suggererait egalement une datation fin me-me s., M.-Th. RaEpsakT-linariak, La datation des inscriptions latines dans les provinces occidenlales de limpire romain dapris les formules " in h(onorem) d(omus) d(ivince) el "deo, deae", dans Aufstieg und Viedergang der röm. Well abrige : A.N.R.W., II, 3, Berlin, 1975, p. 232-282, particulierement, p. 2.40.

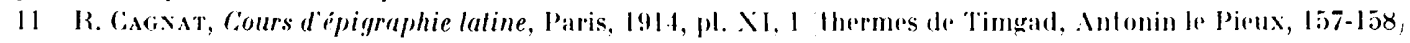

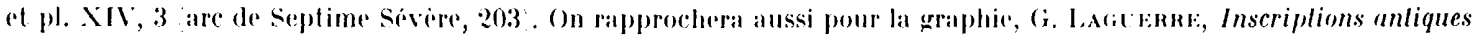

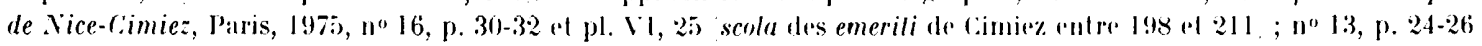
ot pl. VI, 23 inscriptions des thermes dedioes a septime cet raracalla.

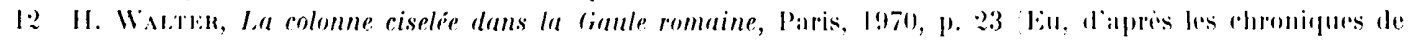
liallial; ; pour la dalalion, p. 39.

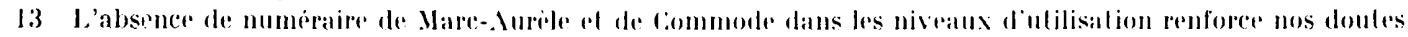

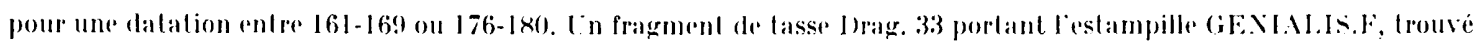

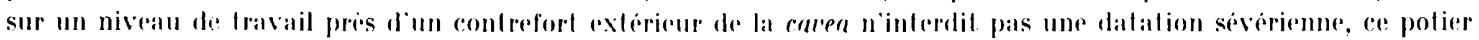

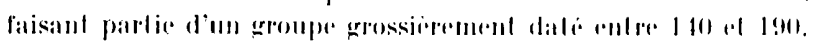

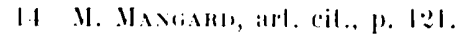

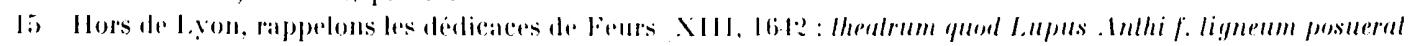

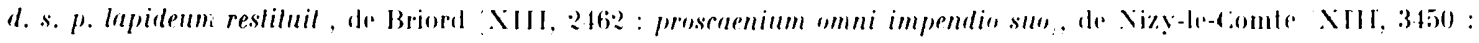



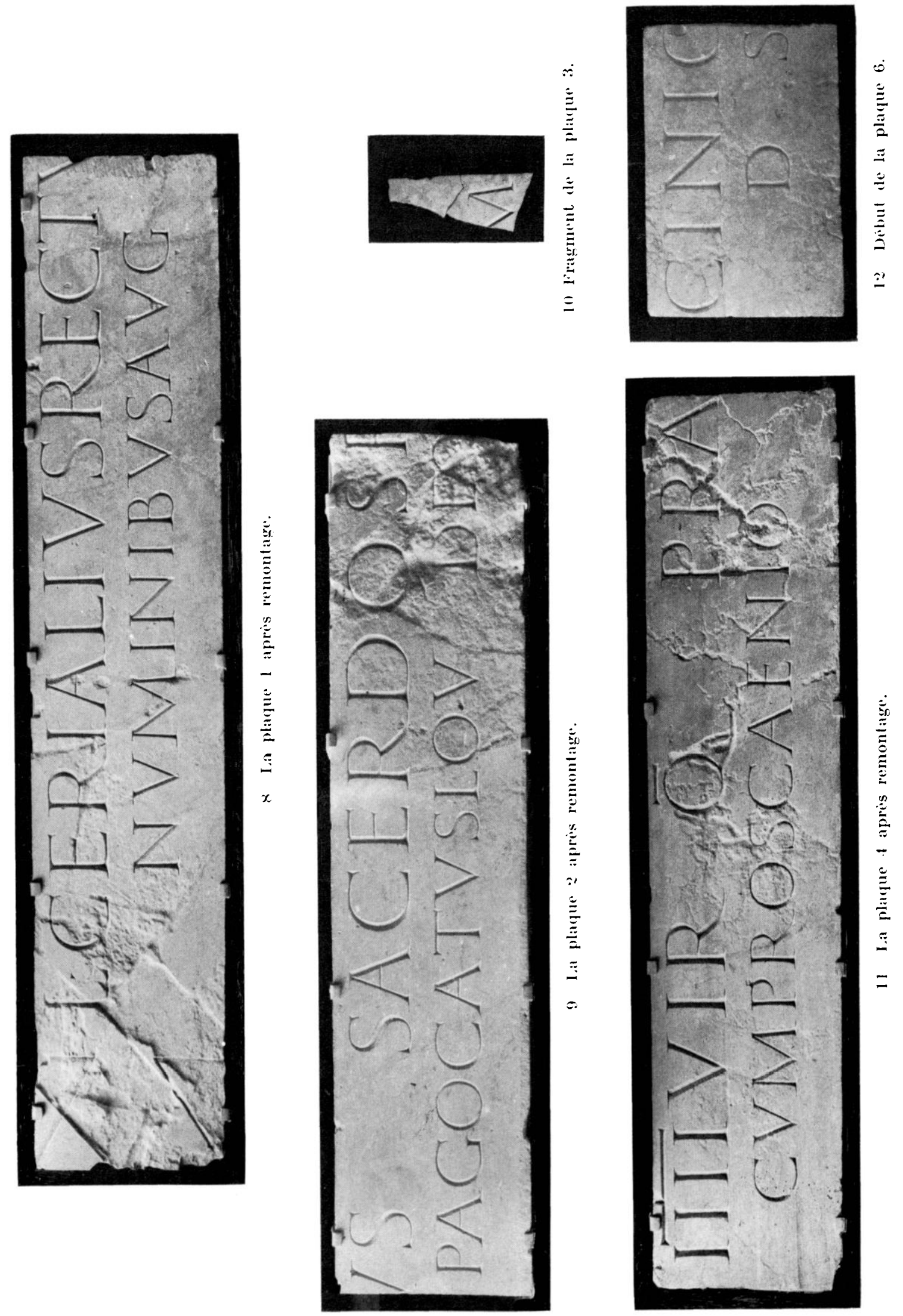
mesure où Cérialius ne s'est pas borné à apporter une simple contribution, mais a financé l'ensemble de la bâtisse et de sa décoration. Il paraît néanmoins avoir visé au plus juste prix : les matériaux (silex, moellons de craie et de tuf, sable, gravier de mer) sont de provenance locale; les proches vallées de l'oise et de la Basse-seine ont fourni les pierres du décor. L'utilisation d'une pente naturelle pour l'assise de la plus grande partie de la cavea a réduit les apports de remblais. La mème recherche du moindre coùt a eu des effets plus fâcheux sur la profondeur des fondations et le volume des maconneries. less colonnes cisclées, couronnées de l'inscription, constituent le seul luxe sur lequel le texte na pas manqué d'attirer l'attention des spectateurs.

Le dédicant porte les tria nomina et possède tres vraisemblablement la citoyenneté romaine acquise au moins per honores comme le montre son cursus. Le nomen en -ius est forgé sur un cognomen selon un usage répandu dans l'onomastique gallo-romaine.16. Le surnom Cerialis qui a servi de base est fréquent aux $\mathrm{II}^{\mathrm{e}}$ et $\mathrm{III}^{\mathrm{e}}$ s. $^{17}$. Par contre, le nomen Cerialius, bien que considéré parfois comme gentilice italien trarlitionne ${ }^{18}$, n'apparait guère qu'en Gaule, et seulement à Lyon, à partir de la seconde moitió du II $^{\mathrm{e}}$ s. ${ }^{19}$. Avec de rares exemples dans la Péninsule et à Rome, le cognomen Reclus n'est pas beaucoup mieux représenté20. A partir de tels indices, l'origine du personnage demeure obscure. De mème. l'absence d'indication touchant la filiation et la tribu ne permet pas de préjuger d'une citoyenneté acquise seulement de frâiche date. En effet, à l'époque de la dédicace, de telles mentions sont pratiquement sorties de l'usage ; au demeurant, hors de la Narbonnaise, la mention de la tribu ne parait pas avoir revetu une grande importaner pour les fiaulois romanisés ${ }^{21}$ et, au début du nue s., elle est à peu près tombée en désuétude.22.

D'après le rang des magistratures, le cursus de Cérialius est présenté dans l'ordre inverse. Dans ces conditions, la fonction remplie avant la questure correspond à une de ces charges municipales subalternes confiées par l'ordo decurionum à divers prafecli²3. Le "préfet à la répression du brigandage " nous est déjà connu par deux inscriptions dans lesquelles le titre apparaît sous des formes plus ou moins abrégées : praefec li] arcend. lalroc[inis] $($ XIII, 5010 = I.L.S. 7007, a Nyon); [praef.] lalr. arc. (XIII, 6211, près de Bingen). Le pluriel lalrocinis n'est pas surement atteste et, 'n l'état du texte, le singulier

proscaenium (...) dono de suo dedil), de Mirebeau (XII, 5614: proscaenium veluslate coruptum (sic) de suo resliluil; et des Tours Mirandes (AE, 1967, 303: thealrum de suo (...) donavit;.

16 II. Forit, Il reclulamento delle legioni da Auguslo a Liocieziano, Rome, l95.3, p. $x 6$.

17 E. Grong et A. Strix, P'rosopographia Imperii Romani, IT, Berlin, 1936, p. 149-150.

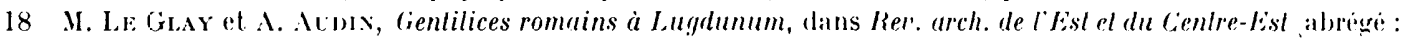
R.A.E., XXIV, 1973, p. 539 et n. 3.

19 A. Atmx of Y. Bunaxn, Chronologie aes apilaphes romaines de I.yon, dans Rev. Eiludes anciennes, 61 , 1959 , р. $320-352$.

20 Le cognomen paraît cependant lié à la zone médilerranéenne $[15,4031$, Pompéi ; V; 7789, Bastia ; X, 2660, Pouzzoles; . Le dédicant de I.J...5. 6953 =: II, 3424, à Carthagene, precise domo Roma; a Rome, VI, f:23.

21 II. Wor.F, Krilerien für lat. und röm. Slädle in Gallien und Germanien und die "V'erfassung" der gallischen Slammesgemeinden, dans Bonner Jahrbücher 176, 1976, p. 90-91 desolmais cite H. WoL.F, Krilerien.

22 G. Rupркант, Inlersuchungen zum Ieliurionensland in den nordwestlichen Provinzen des rim. Reiches, Kallmünz, 1975, p. 110.

23 W. Exsisx, Praefecins, dans R.L. XXII, 2, 1954, col. 13330. 

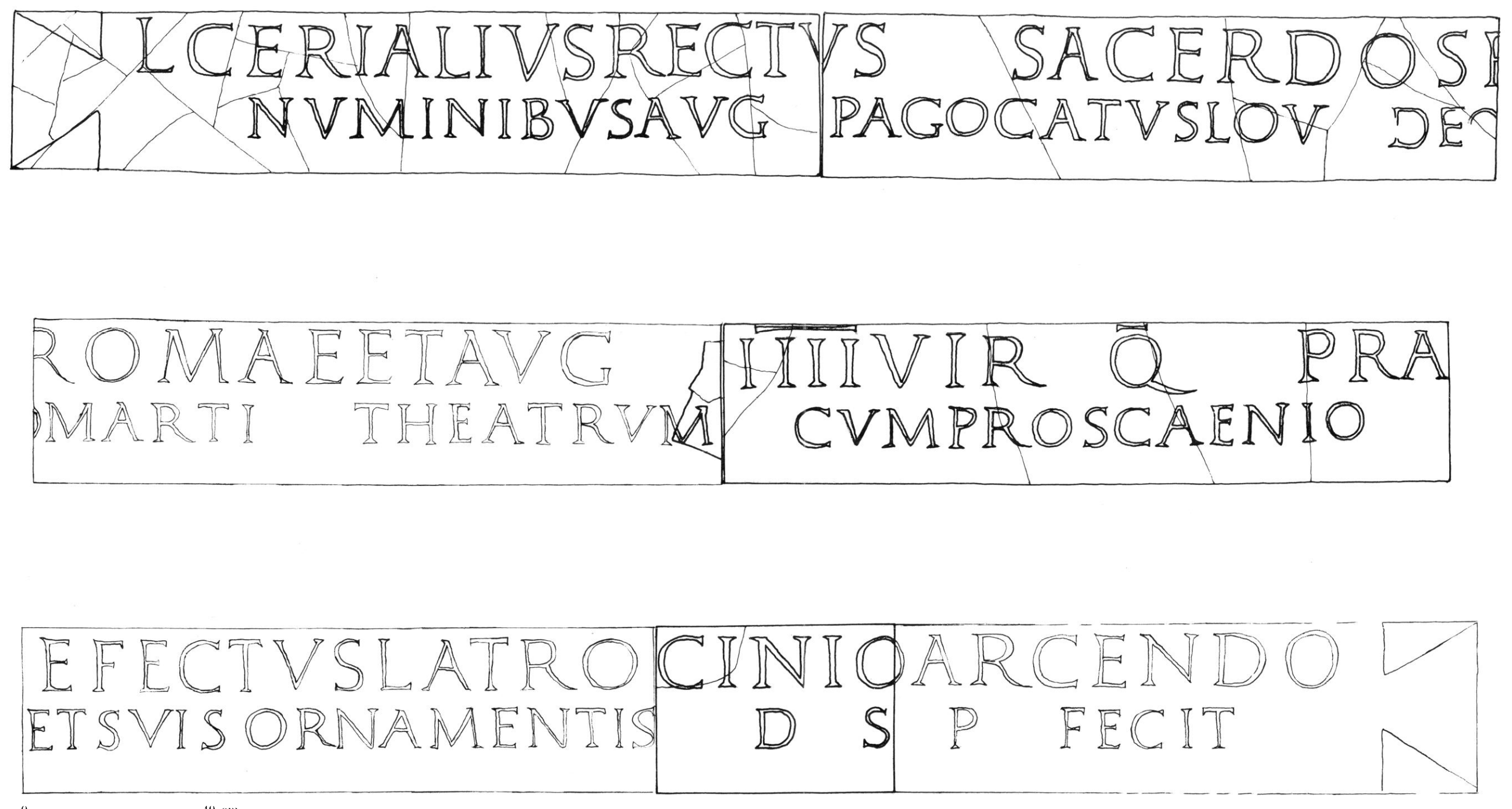
est possible sur l"inscription de Yyon qui présente dans l'ordre direct une carrière municipale menée jusqu au flaminat augustal en passant par le duumvirat géré à deux reprises, la première charge étant la préfecture du brigandage. Celle-ci vient également en tète d'une série de préfectures obtenues par le dédicant de l'inscription trouvée près de Bingen.

L'insécurité commence à se développer en Gaule. en particulier dans le .ord, à partir du règne de Marc-Lurele: sous celui de Commode. Maternus mobilise contre lui les forces militaires de la province. Septime-siévère. déja confronté au problème en qualité de légat de Lyonnaise. intensifiera la répression dans tout l'Empire cont re les lalrones. Le mot ne s'applique pas seulement aux brigands, déserteurs ou hors-li-loi, il désigne également les barbares qui s'attaquent aux frontières du monde romain ${ }^{25}$. Or. dies la fin du II $^{\text {es. }}$ les pirates chauques multiplient les raids sur les rivages de la mer du Yord et de la Manche orientale. Quand on examine les 16 trésors monétaires enfouis entre les estuaires de la Breste et de l'Irques dans une bande coitie profonde d'environ $20 \mathrm{~km}$, on peut déterminer deux périodes privilégiées : la seconde moitié du ir e s. et la fin du rìgne de Commode. Toujours a proximité de la còte. plus vers l'oues, la villa de Colleville près de Fécamp est détruite a la fin du II $_{\mathrm{e}}{ }^{26}$. Ces indices régrionaux de troubles et de destructions, pour ìtre encore peu nombreux dans l'état actuel des recherches, s'inscrivent néanmoins dans un contexte historique bien établi el rendent vraisemblable l'existence de la mission confiée à Cérialius.

S'agissial-il d'une simple police municipale dans l'acception moderne du terme ou plutôt. compte tenu de ce que recouvre le mot latrones, d'une fonction militaire - analogue à notre grendarmerie - en liaison avec une slation si l'organisation d'une ligne douanière sur les còtes de la Manche et de l'Atlantique n'est attestée que par un texte de strabon (IV, 5), 3), le développement ultérieur du trafic entre la Bretagne et les (Gaules n'implique pas, bien au contraire, la disparition du système douanier dans ces zones. En l'étal de nos recherches sur le terrain nous ne saurions aflirmer la présence d'une stalio dans le secteur du Bois l'Abbé dont toutefois la position frontalière apporterait une présomption favorable de plus pour la fonction de Cérialius. En effet, a propos de la frontière entre Ambiens et Calètes, nous avons été amené à établir celle-ci, pour la région qui nous intéresse, au-dessus de la rive droite de l'Yères, ì 7 ou $8 \mathrm{~km}$ au sud/sud-ouest du Bois l'Abbé27, en fondant essentiellement nos conclusions sur l'étude du monnayage gaulois trouvé sur le site ${ }^{28}$ et sur l'analyse des documents ecclésiastiques ${ }^{29}$. Il faut ajouter que la frontière entre civilates devient ainsi frontière provinciale entre Belgique el Lyonnaise puisque Luquste. entre 16 et 13 (?), a rattaché à cette derniere Calites et Véliocasses.

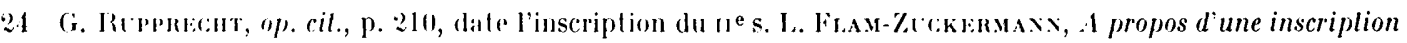
de suisse (C.I.L. XIII, 5010): élude du brigandage dans l'Empire romain, dans Lalomus, XXIX, 1970, p. 451 s., propose une dalalion ${ }_{1} \mathrm{e}^{\mathrm{e}} \mathrm{HI}^{\mathrm{e}}$ s. Pour l'emploi du singulier, Cates. B. (i. VIIT, 24, 3 repentino latrocinio atque impetu enrum eranl oppressi of l'an. Vot. I, 4 (discours d'Eumine) latrocinio Balavicae rebellionis obsessa.

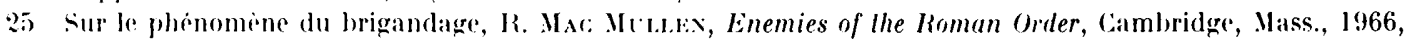

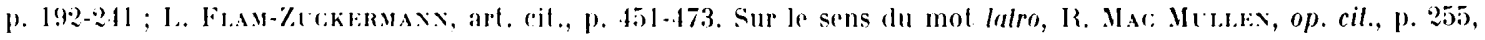

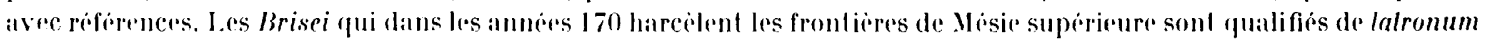
manus dans l'Anné épigraphique abregio : A.E., 1956, 1:4.

26 Sur la date de la destruction de la villa de colleville, ef. R. Soctudivas, dalis fortum, no 3, 1973, p. 34.

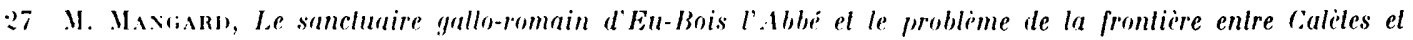

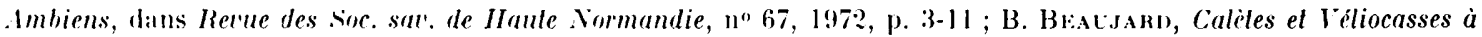
lépoque gallo-romaine, dans Caesarodunum, XVT, 1981 (.Acles du colloque: Fromtiores en (iaule), p. 94-107.

28 L.-P. I) L. monnaies ganthises de Bois l'Ablin, these de 3" cycle, Rernnes, 197.

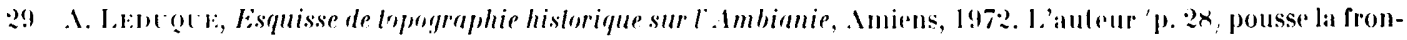

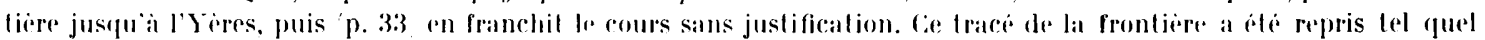

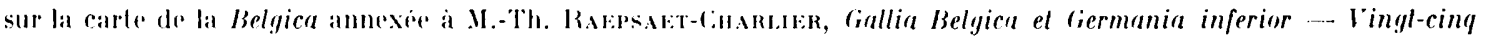

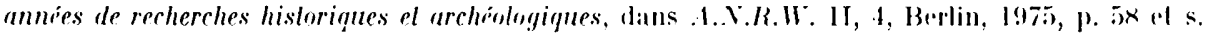




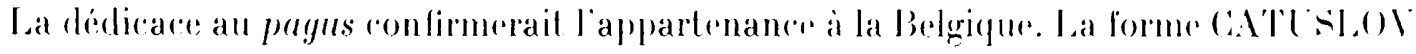
est a rapprocher du nom des Calushugi cites par Pline l'Aneien au nombre des populi du Belgium. Bien que dans les abréviations de toponymes ou de noms de peuples, la coupure apparaisse le plus souvent apres la consonne terminant la racine, il existe des exceptions lorsque te développement ne fait aucun doute pour les destinataires du texte. Le mot pagus étant en règle générale suivi d'un déterminant au mème cas. nous proposerons la lecture pago Caluslou(go). La composition ot le sens du mot formé de *alu- "combat " of de "slougy-o- "armée» ne font pas difficulti"30.

si notre identification est fondée, les Caluslugi oreupaient le terroir connu au ve s. sous le nom de pagus Vimnaus, aujourd'hui le Vimeu, aux marches occidentales de la Picardie. Mais des 27 peuples mentionnés (jusqua aux Helvetes) dans la liste plinienne, une fois retirés les 4 peuples rattachés à la Germanie supérieure (Helvètes, Lingons, Rauraques et séquanes), 14 seulement des 23 restants sont unanimement considérés comme constituant les cités de la (iaule Belgique. I)ans la mème zone cotiere (?), d'autres peuples (oromarsaci, Brilanni, Bassi) restent unal localisés ; leur existence est mème parfois remise en question. Ils auraient en tout cas subi un traitement analogue a celui des Catuslugi, le rattarhement a une civiles, reneontrant un sort inverse de colui qui etait réserve, par exemple, aux Silvanectes peut-itre ériges en cité autonome sous le règne de Claude. Al'époque d'Auguste, Strabon $(\mathrm{IV}, 3,5)$ ne connait en bord de mer, du Rhin a la seine, que les Ménapes, les Worins, les Bellovaques (?), les Ambiens, less Suessions (!) at les Caletes. Tant que demerurent les problèmes posés par la datation des sourees pliniennes, toute hypothèse chronologique éhafaudée sur leurs seules données serait hasardeuse. Notons cependant que R. Fossier, étudiant d'un autre point de vue l'ocerupation de la Picardie a l'epoque gauloise conelut que "la nature tries particulière tant géographique que politique du Ponthien et du Vimen, et qui ne cessera de staffirmer au . Ioyen Age (...), incile a leur donner une individualité. füt-ce en admettant qu'il s'agit d'une peuplade cliente des Ambiani »31. W'autre part, l'étude des monnaies gauloises recueillies an stratigraphie a amené L..-P. Delestrée à conclure que, dans les décennies suivant la conquête, le Bois-l'Abbé "relevait d'une coller'tivité locale jousissant pour le moins à l’époque tardive d'un pouvoir émetteur autonome ${ }^{32}$. Quoi qưil en soit, au début du Ine s., les Caluslugi subsisterairnt romme habitants d'un pagus rlépendant vraisemblablement de la cité des Ambiens.

Comme il est pratiquement de régle dans les Trois Ciaules. la carriere municipale proprement dite de Cérialius souvrira par la questure ${ }^{33}$. IBien qu il ne fasse étal que de deux magistratures, son cursus nest nullement abrégée ; Ir schéma classique -- questure.

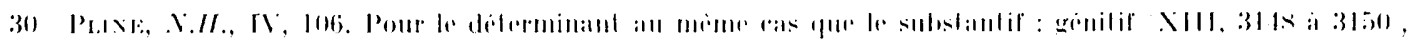

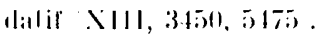

31 R. Fossit: , of, cil., p. 1:25-1:26;

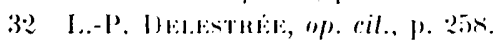

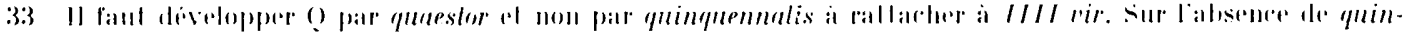

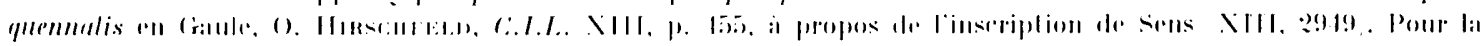

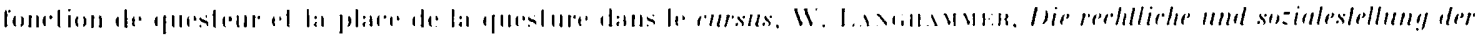

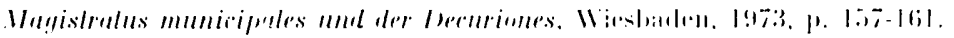

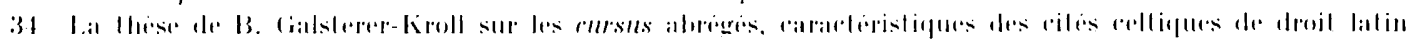

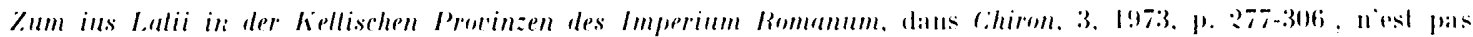

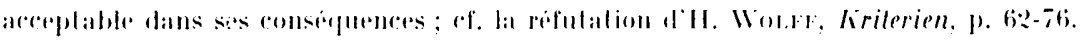


edilite. dumm quattuor-) virat géneralise à partir dexemples italiens ${ }^{35}$ ne se renconter pas dans les Trois Ciaules, hors de la colonie de Lyon par exemple. XIII. 19010). Lin fait, tout se passe comme si les titres de questeur ou d'édile étaient exclusifs l'un de l'autre ou, lorsquils coexistent ainsi en Narbonnaise tenus pour équivalents ${ }^{36}$. Questure of edilité apparaissent l'une et l'autre comme les seules magistratures subalternes, du moins quand elles sont revertues dans le ressort de la ciriles, ce qui est généralement précisé37. Mais clles peurent aussi sexereer dans les carleses plus restreints d'un pagus, d'un ricus, voire d'un convenlus ${ }^{38}$ et alterner alors avere l'autres rharges a raractère essentiellement financier $r^{39}$.

Apres la questure, Cérialius devint quathornir. Le titre est tris rarement porté dans les Trois Caules. Connu che\% les Convenes (I.L.T.G. 76-80), on l'y considere comme ume survivaner de leur ancienne appartenance à la Narbonnaise oì il apparait communément dans les colonies. Le terme existe également rher les séquanes (XIII, 5343) dans une dédicace a Mars Augustr faite par quatre personnages dont un, peut-itre deux, portent Ir titre de qualluorvir. Datée par Hirschfold du a er s., l'insseription remonterait au mème temps que celle (V. 6887) qui mentionne la colonie dont les magistrats suprèmes sont des duumririto. Che\% les ségusiaves, létal du lexte de XIII, 16:4, permet toutes les ronjerlures ${ }^{41}$. Aussi, à la charnière des I $^{\mathrm{e}}$ et II $^{\mathrm{e}}$ s., notre Cérialius fait-il figure d'exerption dans la ciante Cherelue.

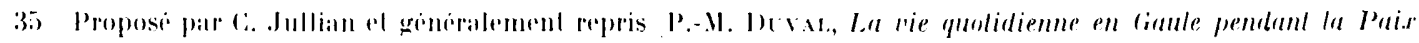

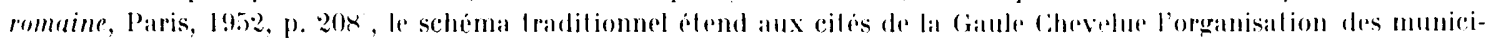

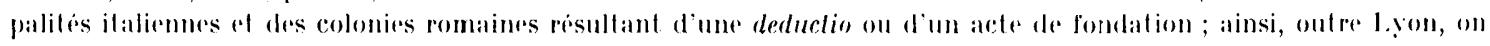

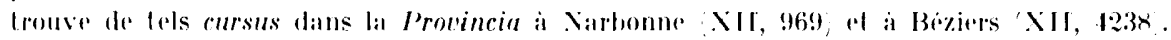

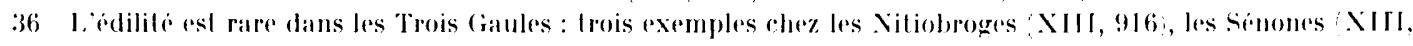

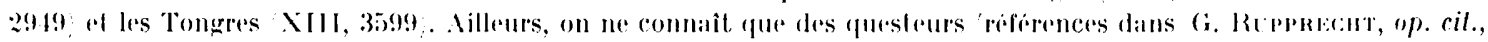

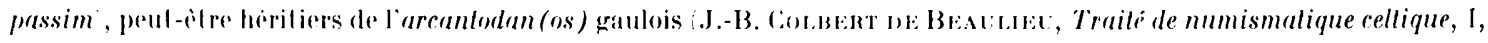

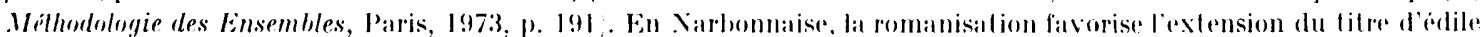

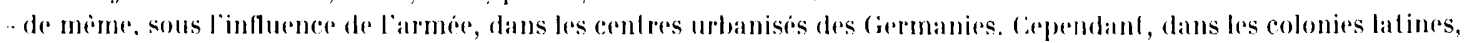

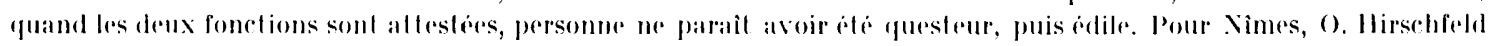

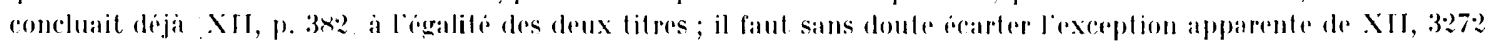

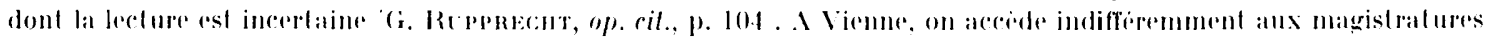

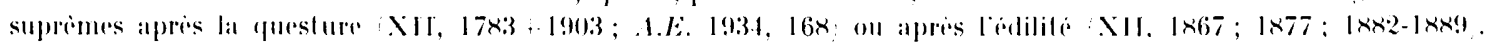

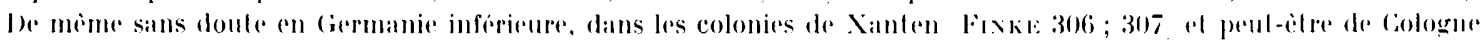

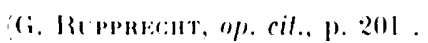

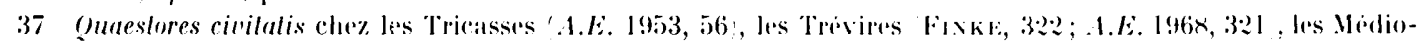

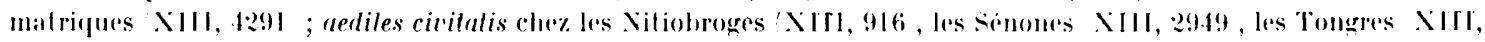

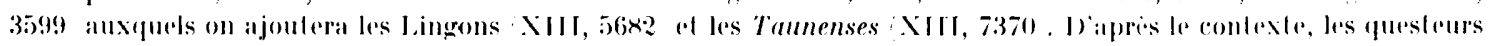

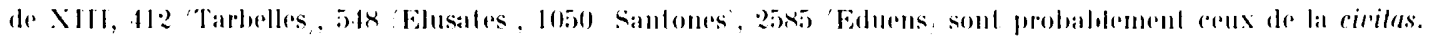

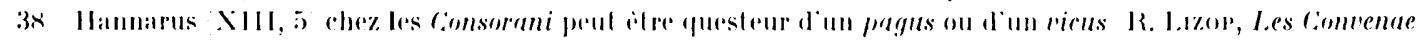

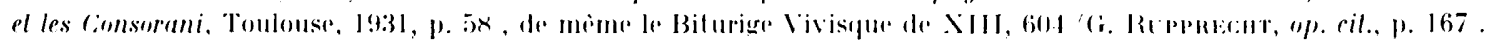

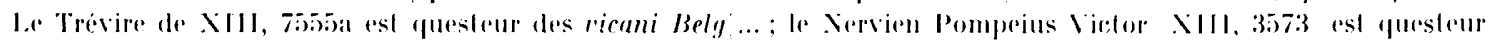

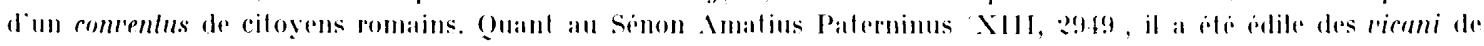

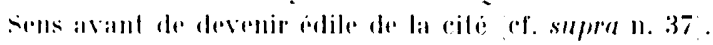

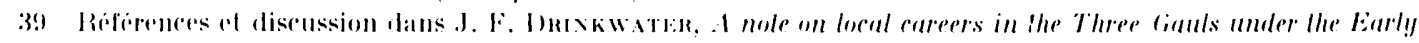
Empire, dans Brilamnia, X. 1979, 11. 92-94.

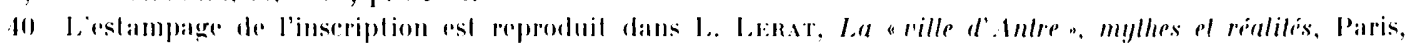

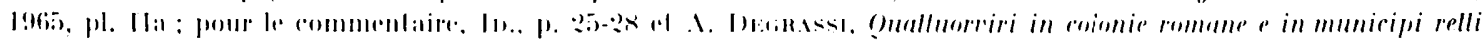

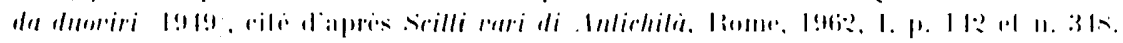

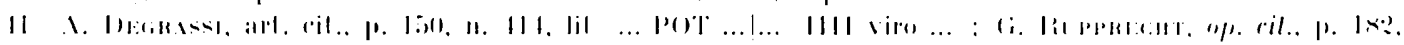

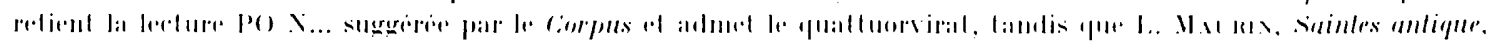

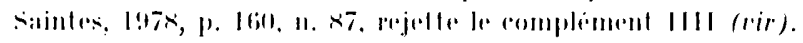


L'étude de la composition des collegges qualtuorviraux en Gaule dans laquelle nous ne pouvons entrer ici. montre que le terme qualluorrir au singulier ne s applique, sauf mention particulière, quau magistrat exereant le pouroir juridictionnel; ce fut certainement le cas de Cérialius.

Qu'il ait atteint la magistrature suprème, la suite de sa carrière couronnée par la prêtrise de Rome et d'August $e^{42}$ nous en apporte une preuve supplémentaire, mème s'il s'agrit, comme nous le pensons, d'une prètrise locale et non pas du sacerdoce du culte fédéral a l'autel de Lyon, bien que, récemment. I. Maurin. reprenant la thise d'H.-(i. Pflaum ${ }^{33}$, ait soutenu que le titre de sacerdos s'appliquerait au seul prètre du culte fédéral ${ }^{44}$. Or. les titulaires du sacerdoce fédéral ont toujours pris soin de le préciser. en particulier hors de Lugrdunum : de même dans les autres provinces de l'Empire, lorsque le prêtre en charge du culte provincial des empereurs porte le titre de sacerdos (Pannonies, Dacie, par exemple). le terme est habituellement complété par une spécification ${ }^{45}$. Quand, au confluent, apparait exceptionnellement une formule abréáée (XIII, 1675) ; I.L.T.G.. 217), le contexte léve toute hésitation. Au demeurant, au fil des siécles, la tendance est plutiot a l'allongement de la titulature, en particulier sous les derniers Antonins el les Sévères ; la formule brève sacerdos Romae el Aug., concevable à la rigueur a Lyon mème. aurait ailleurs dans les (iaules une ambiguïté gènante pour la fierté du prêtre fédéral.

De plus, les prètres fédéraux ne font presque jamais référence de façon précise à leur carrière municipale. Outre la dizaine de cas où pour diverses raisons il n'y en a pas mention, on rencontre le plus souvent une formule-résumé du type honoribus omnibus apud suos functus ${ }^{46}$. La rìgle ne souffre que deux, peut-être trois exceptions (XIII, 1684:1674-1675) et 1717 ?). Il en va lout différemment pour les prêtres locaux. (Onze fois, douze avec notre texle, des éléments du cursus accompagnent le litre sacerdos Rom(ae) el tug. ${ }^{47}$. C'est assurément le caractire local du sarerdoce qui explique ce lien marqué avec les magistralures municipales. I notre avis. hors de Lyon, la litulature courte ne peut concerner que le desservant du culte impérial local.

I)ans la triple dédicace du début de la seconde ligne. la référence aux numina Augustorum associe le culte impérial au pagus consiléré comme entité politique et probablement à la divinité tutélaire de celui-ci. Sans écarter absolument l'hypothèse d'un thényme

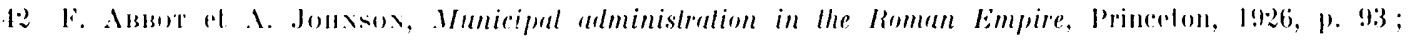

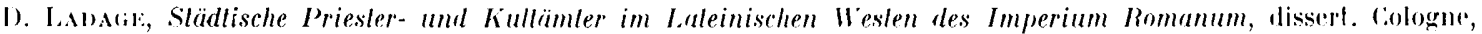
1971 , p. 91. Ses remarques, p. 100, ne s'appliquent ni aux provinces, ni, dans celles-ci, aux prètres du culte impérial. En dernier lieu, J. F. Drovkwater, art. cit., p. 9.4 et n. 31.

43 H.-G. Priacm, Le marbre de Thorigny, Paris, 19.4x, p. 13. Point de vue adoptio encore par H. Wolff, Civilas und Colonia Treverortum, dans Historia, XXY, 1977, p. 207-209. - Publiant les fextes de Saint-Bertrand-

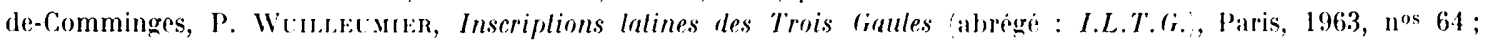
76-80; 82-8.1 et p. 26, se Pallie a l'opinion d'A. Aymard Kev. Éludes anciennes, 19.11, p. 216-229) qui avait soutenu que la formule simple s'applique a un pritre du culte municipal. Avee queleque hésilation, J. Bousquet parail parta-

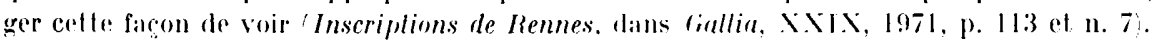

4.4 I. . Matris, op. cil., p. | |x|-201.

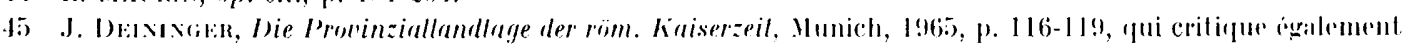
P(1:aum : (p. 101, n. 7 .

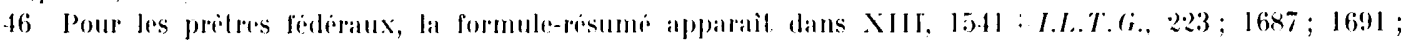
$1694 ; 1698+I . L . T .(6 ., 220 ; 1702 ; 1710 ; 1714 ; 1718 ; 1719 ; 29.40$ a ; 3162; 5353-1.E., 1965, 341 ; 11174; A.E. 1952,

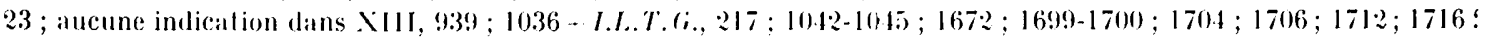

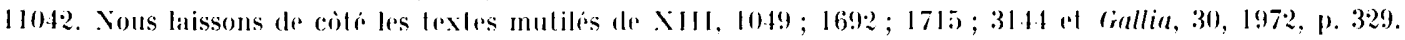

17 lour les prefers locaux, les indicalions fouchant les magistralures municipales appataissent dans Xllf,

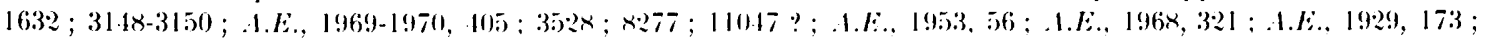

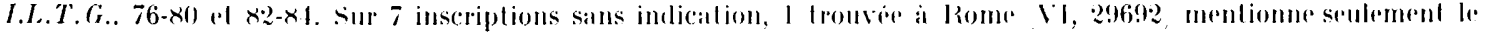

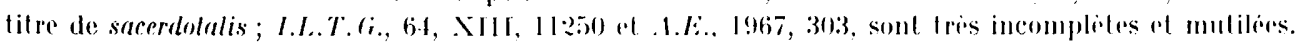


celtique, impossible a déterminer, nous pencherons plutot pour un Vars gallo-romain. avatar d'une divinité indigène. non pas en rapprochant un peu facilement le dieu de la guerre du nom des Caluslugi. mais en considérant l'extension d'un culte dont la prééminence s'explique par l’assimilation d'un grand nombre de divinités locales. Leur association au culte impérial est par ailleurs bien attestée no re rapport fréquemment exprimé confère a la pretrise de Rome of d'Auguste. au-dela de sa fonction religieuse, une valeur politique qui l'emporte en prestige et autorite sur les titres juridien-arministratifs de la rarrière antérieurement parcourue.

Dans le cas de Cérialius, cette arrière soulexe ancore deux questions. Quel était le statut de la communauté dont les magistrats suprémes portaient le titre de qualluorvir? Dans quelle agrglomération se trouvail le siège de la magistrature de Cérialius ?

Vers la fin du II $^{\mathrm{e}}$ s.. les magistrats des municipes italiens ou provinciaux el des colonies succédant ou non is un municipe portent en riggle générale le titre de dummeir. Celui de qualluorrir peut. par exception, soit persister dans quelques colonies dont nous savons qu'elles sont issues d'un municipe, soit apparaitre occasionnellement dans quelques municipes fondés à partir d'IIadrien ${ }^{49}$. Nous pouvons donc nous demander si le rapport primitif existant entre qualtuorvirat et municipe avait jamais cessé d'être perçu et n'aurait pas influencé le choix de l'administration impériale.

Suggérer que Córialius aurail élé quathorrir d'un municipe de Gaule Belgique se heurte à une difficulté majeure : l'absence de municipe attesté expressément en Gaule Chevelue. Absence que l'on explique par les caractires originaux de la civilas gauloise. lesquels entraineraient la propagation privilégiée du titre colonial dans le domaine celtique proprement dit ${ }^{50}$. Les deux ou trois municipes connues en Germanie ne contredisent pats cette explication ${ }^{51}$. Il s'en faut pourtant que l'ancienne aire de l'expansion reltique ignore totalement le municipe. En Bretagne, che\% les Caluvellauni. Tacite (Am. XIV, 3:3) mentionne le municipe de l'erulamium. (On n'a certes pas manqué d'observer que l'historien emploie souvent re mot pour désigner une ville sans connotalion juridique particulière, guidé parfois par des considérations stylistiques. Cependant. dans le contexte visé, Tacile, écrivain mais aussi fonctionnaire au fait des institutions. cite en l'espare de deux pages Irois villes bretonnes: Colchester (XIV.31 : in coloniam Camulodunum). Londres (XIV, 33) : Londinium (...) cognomento quidem coloniue non insigne) et T'erulamium (ibid.: municipio l'erulamio) en prenant soin a chaque fois de préciser le statut qu'elles ont ou qui fait defaut : le choix des mots colonia ou municipium ne répond pas ici a une exigence de slyle ${ }^{52}$. Ln Narbonnaise, le décret de Iligne (.I.E.. 1961, 156) nous a révélé sous Commode l'existence chez les Bordionlici d'un municipe qui ne peut remonter au-deli d'Ifadrien ; notons que le titre des magistrats n'est pas assuré. la plaque de bronze laissant subsister une large lacune devant ...V IR I53. Expliquer ce municipe incongru par l'ancienne

Is A. Cuastariot. L'organisation du culle impérial dans la cilé à la lumirre des inscriplions de Rennes, dans

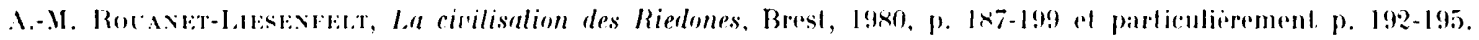

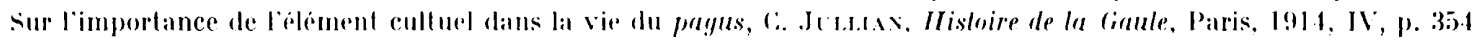

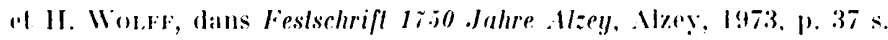

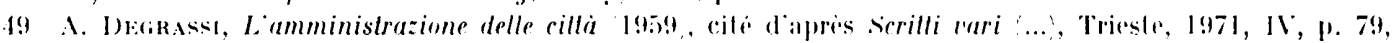
reprenant la these developpere dans la publication citeresura n. Al. tui fail remonter les qualluorviri des colonies atux

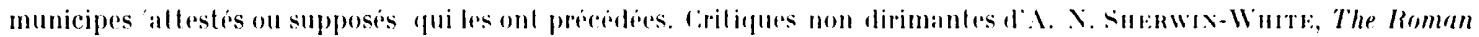
Cilizenship, oxford, :2e id., 1973, p. 365, n. 1.

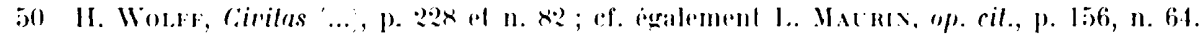

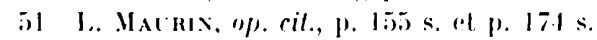

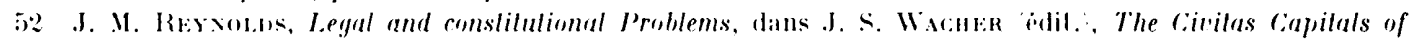

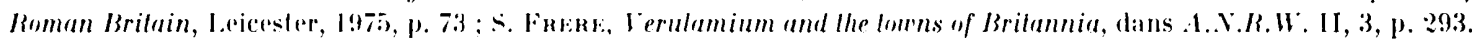

53 H. Hollaxu, Inscriplion sur bronse de Thoard, dans Gallia, XVIII, 1960, p. 10.4-105, fig. 1. 


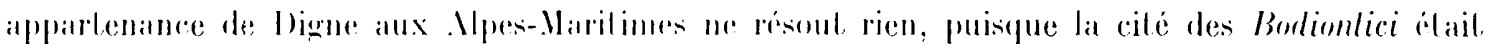

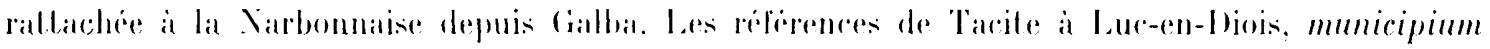

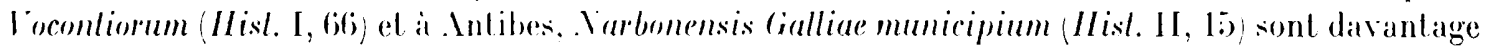
sujetles a caution. Lexistence d'un qualluorrir (XII, 176) et la tribu Voltinia rapprocheraient plutot Anlipolis des colonies latines de la l'rovincia, cependant on y trouve au se s. un duumvir (XII, 175) comme dans les municipes voisins d'Italie et des Mpes-. Maritimes ${ }^{54}$. Ians les provinces alpines, l'institution de municipes est mieux assurée. A Cimie\%, la dédicace d'un autel (A.E., 1965), 193) aux desservants du (iénie du Municipe, dalée du e $^{\mathrm{er}}$ s., suit sans doute de peu la concession du droit latin par Néron aux peuples des IJes-Varitimes Tar. Amn. XV, 32); les magistrals ronnus

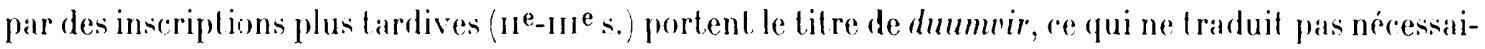
rement une modification du statut ${ }^{55}$, a en juger par les magistrats des municipes flaviens (? $)^{56}$ de Briançon (XII, 95) et de suse (V, 72:35) dans les Alpes Coltiennes qui grardent le titre de dummer généralisé à la mème époque dans les municipes d'Espagne.

Ainsi dans le domaine rellique nous aurions trois municipes dans les provinces alpines a partir du rigne de Véron et sous les Flaviens (un probable dans le mème lemps en Bretagne) dirigés par des duumviri; un, peut-ètre deux, en Narbonnaise constilués à une époque voisine de celle où sont. créé dans les provinces danubiennes ceux ayant a leur têle des qualluorviri, à partir du regne d'Madrien. Pour être maigre, le bilan n'est toutefois pas nul et. si nous reportons notre altention vers la Gaule Ghevelue pendant les trois premiers siecles. nous ne pouvons faire totalement abstrartion de l'insuffisance numérique des donnces fournies par les auteurs ou les inscriptions.

le titre des magistrals presque exclusivement des dummeri -- esl connu pour 10 ou 12 cités sur 21 en Aquilaine (Consorani, Coneenae. Tarbelli, Ausci, Elusales, P'etrucorii, Sanlones, Lemocices. Bituriges Cubi, Vellavi, peut-ître Arverni et I'iclones), 5 sur 26 en Lyonnaise auxquels on ajoutera les lingons et les séquanes (Segusiavi, Ledui, Senones, Viducasses, Riedones) et :3 seulement sur 14 en Belgique, sans compter notre texte (Treveri. Vervii, Morini). I notre avis, le hasard des découvertes n'explique pas seul l'inégalité de cette répartition ; elle traduit plus certainement le degré de: romanisation des institutions publiques. Xous remarquerons qu'en Gaule Belgique, 2 des 3 aités a dummeiri sont altestées comme colonies, de mème en Lyonnaise pour 3 ou plutol 4 cités sur :) (plus encore une fois les Lingons el les séquanes) el en Lquil aine pour 1 sur 10 ou 12: dans cetle province. en admetlant la relation entre flaminal et statul colonial, on ajouterait 1 rilés 57 . Ainsi sur 20 (y compris les Lingons et les séquanes) ou 22 cités gouvernées par des duumviri. 10 probablement 14 - sont des colonies. Nous ignorons le tilre porté chez les Médiomatriques et les Éburovices oi le stalut colonial est conjecturable ${ }^{58}$. En revanche, comple tenu de la généralisalion du titre lant. dans les colonies que dans les municipes, nous hésilons à faire de la présence de duambiri un aulre indice de l'élévation d'une cité au rangr colonial. Mème en franchissant le pas, nous ne dépasserions pas 24 colonies pour plus de 60 cilés. Quand on compare celte situation avec celle de la Narbonnaise ou des provinces africaines, la prolifération du titre colonial reste toule relative, d'aulant plus que dans la moilié des cas, un tel statul demeure une hypothese seulement vrasemblable. Nous retiendrons done le fait qu'un tiers environ des cités des 'Trois Giaules présente des magistrats suprêmes de titre romain, apparus, dans la mesure ou les lextes sont dalables, au cours de trois siecles. Celle constatation nous paraît contredire l'hypothese touchant l'existence possible de duum(qualluor) riri

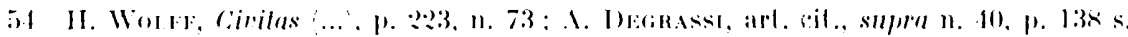

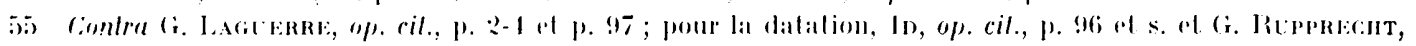
op. ril., 1. 1 1.2-146.

56 B. Galstekth, art. cil., p. 2X6, n. 10.

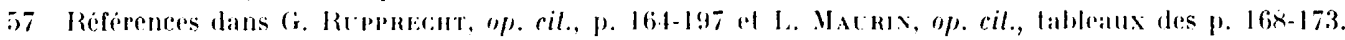

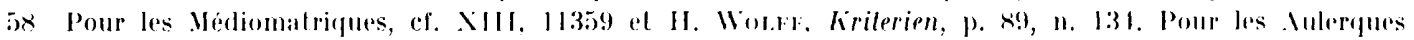
Ehmovices, on rappollera l'exislence d'un flamen XIII, 3:300\%. 


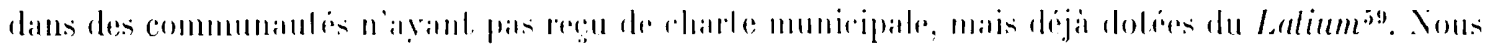

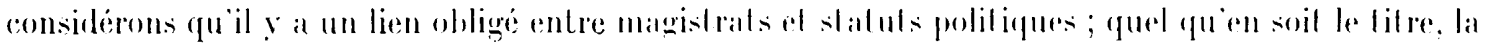
magistrature sexere dans une colonie on un municipe.

A. Chastagnol siest étonné de ne pas trouver de plus nombremses mentions de municipes

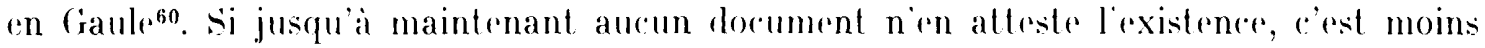
une conception de la civiles dans laquelle rien en fait ni en droit ne sopposait a une telle constitution que lo hasard des décomvertes épigraphiques qui nous parait celte fois devoir etre mis en causeis. Nous ferons observer, par exemple, qüil faut attendre le discours d'Eumene ( $(5,5)$ pour avoir mention de la colonir admise chez les Esluens par la plupart des historiens au moins a partir du a er. Mais que dire des Bemes, amis du peuple romain, liés a Rome par un foedus Pline, N.II. IV. lo6) comme les Eduens dont le chef-lieu abrite le gouverneur provincial de Belgique ot sur lo slatut politique desquels l’épigraphice est muctte Meme constat pour lillustre cité des Carnutes, egalement fédérée et représentée a l'Autel de Lyon. Nous devons de eonnaile la colonie des Morins à une inseription trouvé pres de Vimegue (XIII. 87.27j et celle des sépuanes a une dédieace provenant du Cirand-saint-Bernard (I, 6887). Mis a part les Trevires et les Lingons pour lesquels la doceumentation est relativement abondante, nous disposons seulement de six ou sept textes

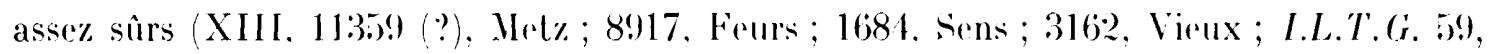
saint-Bertrand-ele-Comminges ; 1577, Le Puy ; 5)6, Eauze) faisant état du statut politique d'une cité. On ne pourra guere ajouter à la documentation sur le sujet qu'une trentaine de textes plus ou moins fragmentes, inégalement utilisables, le plus souvent mal dates ou inclatables, mentionnant les doumeirio2.

A partir de donnés aussi lacunaires sans doule serail-il plus sage dans le cas de Cérialius de conclure a un non liquet. Cependant. considérant d'une part le lien historique entre qualluorviral el municipe et d'autre part l'insulfisance des objections opposées a la créalion de municipes en (iaule. nous proposons de voir dans notre personnage un quallumrer iure dicundo d'un municipe constitue au chef-lieu d'une rité bénéficiant du Lalium. laquelle ne saurail ibre que relle des Ambiani aver.

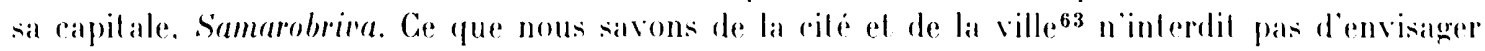
rette promolion que. par l'analogie du litre de Cépialius avere celui porte dans certains municipes damubiens, nous daterions volontiers du rigne d'Hadrien.

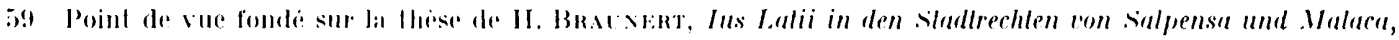

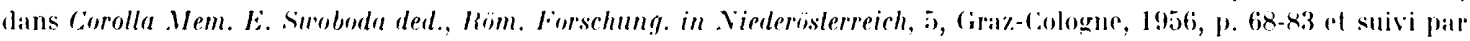

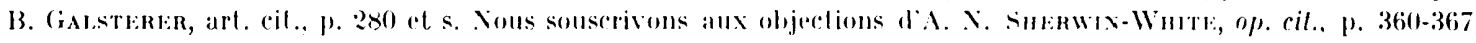

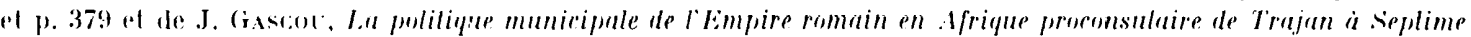

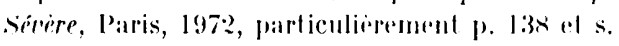

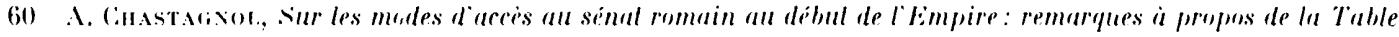

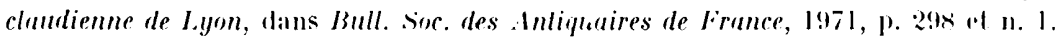

61 Cf. la remarque de l.. Maurin a propos de saintes op. cil., p. 175.

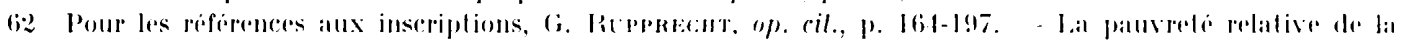
documentation, si elle ne sexplique pas entidement par les hasards et les comblitions de la recherehe, incilerait a

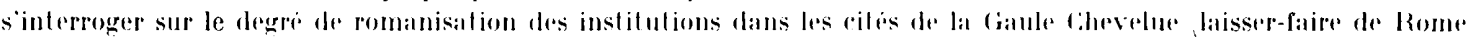
on mangue datimance de la part des danulois ?'.

6.3 F. Wid., Recherches sur le dóneloppement urbuin sous lEmpire romain dans le . Nord de la France, dans

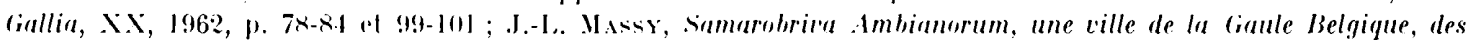

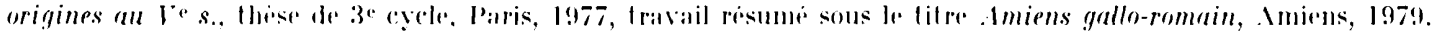


Les Ambiani, au même chef que les aulres peuples coliers ... at notamment les Morini - jouaient un ròle important pour la sécurité des liaisons maritimes ot terrestres avec la Bretagne. I) ce point de vur la répression des lalrones au sens large du terme devait être une des grandes préoccupations de la civilas. Mission importante, on concoit qu'elle ait pu ouvrir la voie des honneurs à ceux qui s'en acquittaient. Exercée dans le carle de la cité. la "préfecture» avait sans doute plusieurs points dapplication et le Bois l'Abbé pouvait être l'un d'eux. Ce qui expliquerait l'interèt manifesté comme évergite par Cérialius Rectus, parvenu au faîte des honneurs, au sanctuaire clu pagus ${ }^{64}$. Son aceession à la prêtrise de Rome et d'Auguste constituait un motif supplémentaire, compte tenu des liens unissant le culte impérial à celui des divinités tutélaires des pagi.

En l'état des recherches. les vestiges connus s'étendent sur plus de 30 ha et l'ensemble apparait comme le plus important du pagus. I a fonction avant tout religieuse du pagus, au moins sous l'Empire, la triple dédicace de l'inscription du thécitre. l'existence d'un complexe d'édifices cultuels ${ }^{65}$ nous incitent à considérer le Bois l'Abé comme le chef-lieu des Caluslugi. Sur la véritable nature de ce chef-lien et ses rapports avec le capul civitatis, nous demeurons réduits aux conjectures. Pour le moment, nous connaissons les éléments habituels d'un sanctuaire. Si l'on ajoute la position frontalière, un peu à l'écart du réseau routier antique tel qu il est reconstituéc6 cel l'environnement forestier qui pourrait remonter à l'Antiquitéb. on y retroure les caractéristiques du conciliabulum selon la définition de G.-Ch. Picard ${ }^{68}$. A titre d'hypothese de travail, nous admettrons rette identification par commodité, tant qu'elle ne sera pas remise en question par les progress de la fouille ${ }^{69}$.

Celle-ci, sur le chantier du grand temple, a etabli que la premiere occupation du site est postérieure à la conquête; elle s̀y est manifestée essentiellement par une série de dépoits

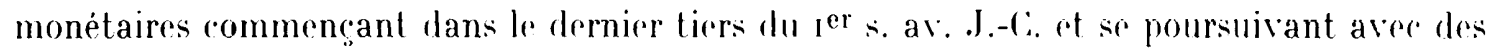
interruptions probables jusquau début du regne de Tibere. sinon de Claude ${ }^{70}$. L'analyse du monnayage gaulois révèle la permanence et la variété l'émissions régionales et locales qui, au moins sous le règne d'Auguste, portent témoignage non seulement de la prospérité

64 En depit des riserves de (r. Rupprecht top. cil., p. xt it s. et n. 7.1-75), nous ne pouvons tolialement axelure que Cérialius soit un ancien militaire que son experienee pendail aphe a lexercice d'une mission de police; l'absence d'élements typiquement indigenes dans ses tria nominn en ferait un "horsain "vemu s"inslaller en lerritoire ambien.

65 .I. MAN(iARJ, art. cit. supra n. 1, p. 128.

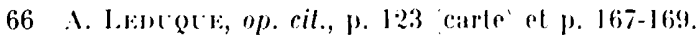

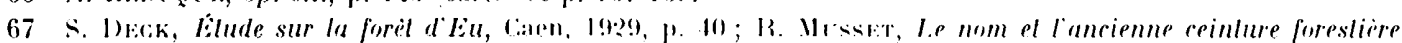
du Pays de Cound, dans Norois, VIII, 1961, p. 3:21-33:7.

68 G.-th. Prcano, Obsertalions sur la condilion des populalions rurales dons l Empire romain, en lianle el en

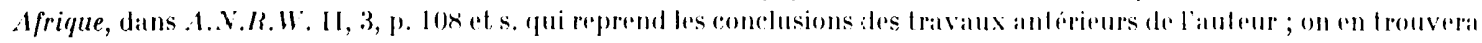

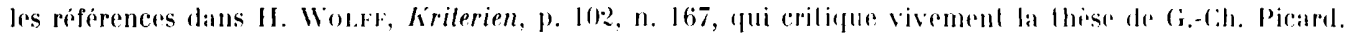

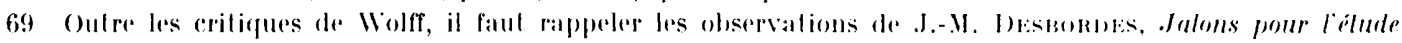

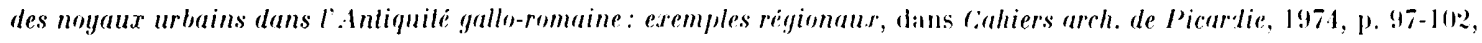
particulièrement p. 102, qui insiste sur le caraction non centralise de la cile ganloise. Io meme, H. Wolff oppose a

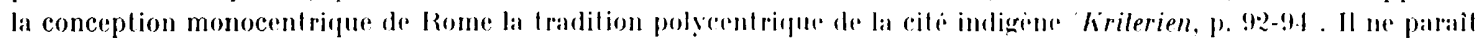
pas cependant, en l'ilat de la documentation, guiun de ces noyanx urbains hormis les chefs-lienx ait jamais

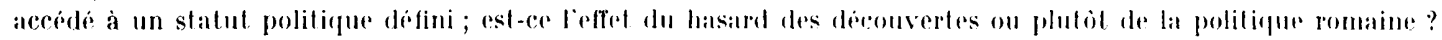

70 M. MAN(iaRD, art. cit. supra, n. 1, p. 1:4. 
maintenue des Ambiani, mais aussi de la vitalité économique, voire de l'autonomie monétaire du peuple des Caluslugin

Rapprochés des conclusions de l'étude numismatique, l'inscription dédicatoire du théâtre et le cursus de Cérialius suggèrent des évolutions liées aux décisions de l'administration impériale face aux problèmes posés par l'organisation politique des Trois Gaules et par l'existence des cités gauloises. Les possibilités d'application du processus d'urbanisation paraissent avoir joué un rôle au moins aussi déterminant que l'intégration au nouveau réseau routier ${ }^{72}$. La prééminence des chefs-lieux à partir du $\mathrm{IV}^{\mathrm{e}}$ s. résulte probablement moins d'accidents historiques comme les invasions que d'une politique plus ou moins ouvertement suivie et visant à substituer à une cité gauloise polynucléaire de véritables circonscriptions administratives monocentriques et hiérarchisées, conformes au schéma romain.

\section{Michel Maxgard}

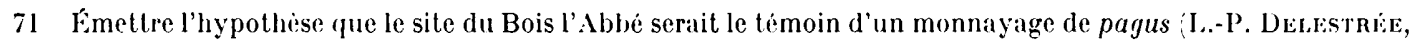
op. cil., p. 244-258 et M. Mavgard, Apporls de l'Archéologie à la Numismatique, dans Monnaies, Médailles el Jelons, catalogue de l'expo. organisée au Musée départ. des Antiquités, Rouen, 1978, p. 71-103) va à l'encontre des idées recues. La proposition est moins choquante si l'on admet que dans les décennies suivant la conquète, les Caluslugi jouirent d'une certaine autonomie au moins jusqu'aux mesures de 16-13 av. J.-C. Au demeurant, la pénurie chronique de numéraire romain laissait place non seulement à la circulation des espéces gauloises, mais aussi à leur émission. Par nécessité, la politique d'Auguste en ce domaine fut sans doute an début du règne plus libérale que celle de Tibère qui òta le ius metallorum aux cités (Suet. Tib. 49), non sans graves conséquences économirques et sociales (A. Gravirr, Tibère el la Gaule, dans Rev. Éludes iatines, XIV, 1936, p. 373-388;.

72 E. Wut.t, art. cil., p. 99. 